FEV1 (\% predicted) between 18-21 years. While there was some evidence of special cause variation (at 2SD limits) in prior to case-mix adjustment, after adjustment none were outside the 2SD limits. None of the centres were outside the 3SD limits in either analysis.

Conclusion In conclusion the work to-date illustrates that funnel plots can be used to explore potential differences in $\mathrm{FEV}_{1}$ between specialist centres. Case-mix adjustment models should develop into a useful tool for making centre comparisons which can continue to be used by stakeholders. This is early work, however, and we need to bear in mind that by examining outcomes in small populations risk missing true differences due to low statistical power. Further work is required to assess whether any observed differences are due to chance or are related to the care patients receive.

\section{P221 EVALUATION OF EXACERBATION FREQUENCY AND RE- HOSPITALISATION, AND RISK FOR SUBSEQUENT EXACERBATIONS IN ASTHMA PATIENTS IN A UK PRIMARY CARE SETTING}

RY Suruki, JB Daugherty, N Boudiaf, FC Albers. GlaxoSmithKline, Stockley Park, UK

\subsection{6/thoraxjnl-2015-207770.357}

Introduction and objective Exacerbations occur in all asthma patients but disproportionately impact those with more severe disease. This study aimed to describe the frequency of exacerbations by asthma severity and the risk for future exacerbation. Methods In a retrospective cohort of asthma patients in the CPRD (2009-2011), we defined asthma severity based on asthma medication use and exacerbation history; index date was asthma medical code date. Asthma severity was determined by asthma medication use and exacerbation history during the 12 months preceding the index date. Exacerbations were ascertained during the 12-month follow-up period and were defined as an asthma-related accident and emergency (A\&E) department visit or hospitalisation, or any oral corticosteroid (OCS) prescription with an asthma medical code recorded within \pm 2 weeks. A proportional hazard model was developed to evaluate the risk for subsequent exacerbations associated with the type of exacerbation (OCS vs. ED/hospitalisation).

Results A total of 211,807 patients with asthma were identified in CPRD during the study period. The mean age was 45 years and females made up $58 \%$ of the study population. Of these patients, $17,785(8.4 \%)$ and $3,592(1.7 \%)$ experienced $\geq 1$ and $\geq 2$ exacerbations, respectively during the follow-up period. The proportion of patients experiencing $\geq 1$ or $\geq 2$ exacerbations increased with severity and prior exacerbation frequency (Table 1). Among 1,900 patients with an asthma-related hospitalisation, $2.3 \%, 3.3 \%$, and $3.8 \%$ experienced asthma-related readmissions within 30-, 60-, and 90-days, respectively. When limited to patients with more severe disease, the readmission rates increased significantly, up to three times in those with a history of $\geq 2$ exacerbations. Compared to an OCS defined exacerbation, an A\&E visit or hospital admission was associated with a $30 \%$ greater risk for any subsequent exacerbation $(\mathrm{HR}=1.3$, 95\% CI 1.15-1.44), after adjusting for gender, disease severity, atopy, and exacerbation history.

Conclusion Asthma exacerbations remain a burden for patients with severe asthma or a history of frequent exacerbations. Additionally, patients should be managed carefully after an asthma exacerbation as they are at higher risk for subsequent exacerbations and readmission. (GSK-funded; WEUSKOP7092).

\section{P222 RATES OF HOSPITALISATION AFTER DIAGNOSIS OF LUNG CANCER: A LINKED AUDIT AND HOSPITAL EPISODE STATISTICS STUDY}

A Khakwani, RB Hubbard, L Tata. Division of Epidemiology and Public Health, University of Nottingham, Nottingham, UK

\subsection{6/thoraxjnl-2015-207770.358}

Much of the research in lung cancer is concerning survival or treatment rates and little is known about the rate of hospitalisation (emergency/elective) following the diagnosis of lung cancer.

Newly diagnosed English patients from the National Lung Cancer Audit database (NLCA), 2007-2011, were linked with Hospital Episode Statistics (HES) data to provide details on their subsequent hospital admissions. Hospitalisations for receipt of chemotherapy or cardiothoracic surgery were excluded to ensure only non-treatment related admissions were included. We only included patients included who survived at least 30 days after their first presentation to a physician to exclude more advanced disease. We calculated rates and rate ratios (RR) of elective and emergency admissions per person-year (ppy) by patient features including sex, age, performance status and co-morbidity.

Among 92,482 patients, there were 261,121 non-treatment related hospitalisations with rate of 2.92 admissions ppy $(95 \%$ CI, 2.91-2.93). Emergency admissions constituted 57\% of all admissions at a rate of 1.66 admissions ppy while the elective admission rate was lower at 1.26 admission ppy. Adjusted RRs in Table 1 show that males were approximately $20 \%$ more likely than females to be admitted through either route (RR 1.17, 95\% CI $1.16-1.18$ for emergency and 1.20 (1.18-1.21) for elective). Worsening performance status, co-morbidity and advanced stage were all associated with higher emergency admissions while there was no strong association with age. For elective admissions pattern were similar yet associations were weaker and performance status did not show a linear association with admissions. Increasing socioeconomic deprivation was associated with a moderate increase emergency admission rates but a decrease elective admission rates.

The rate of emergency admissions was higher than the rate of elective admissions following diagnosis of lung cancer. Sex, worsening performance status, advanced stage and co-morbidity were all independently associated with admissions with similar patterns for emergency and elective admissions. However, being from a more deprived socioeconomic class was associated with more emergency admissions and fewer elective admissions. Reason for these findings could be related to variation in receiving treatment in these groups or treatment related side effects leading to more emergency admissions.

\section{P223 VALIDITY AND INTERPRETATION OF SPIROMETRY FOR PATIENTS IN PRIMARY CARE}

${ }^{1} \mathrm{KJ}$ Rothnie, ${ }^{2} \mathrm{H}$ Mullerova, ${ }^{3} \mathrm{H}$ Goss, ${ }^{3}$ I Chandan, ${ }^{1} \mathrm{JK}$ Quint. ${ }^{1}$ Respiratory Epidemiology, Occupational Medicine and Public Health, National Heart and Lung Institute, Imperial College London, London, UK; ${ }^{2}$ Respiratory Epidemiology, GlaxoSmithKline R\&D, Uxbridge, UK; ${ }^{3}$ Medical School, Faculty of Medical Sciences, University College London, London, UK

\subsection{6/thoraxinl-2015-207770.359}

Background Previous studies have questioned the validity and interpretation of spirometry undertaken in primary care. Knowing that data are accurate is important as many respiratory diseases are diagnosed and managed in primary care. Additionally researchers use data entered into electronic health records both 